
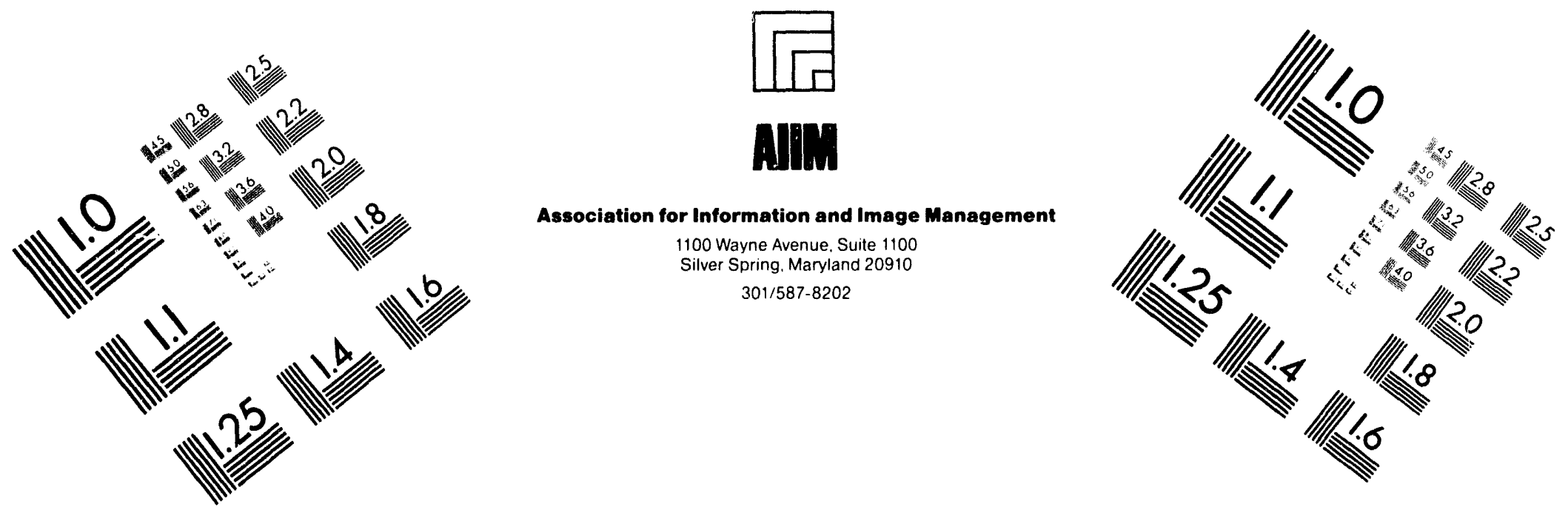

\title{
Centimeter
}

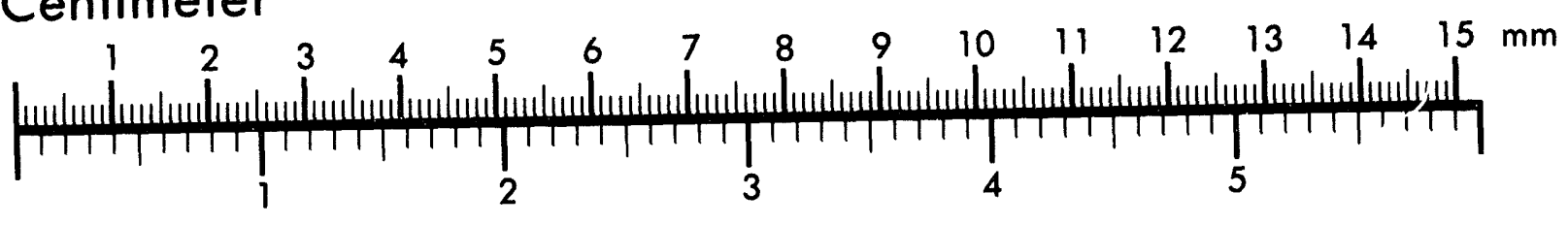
Inches
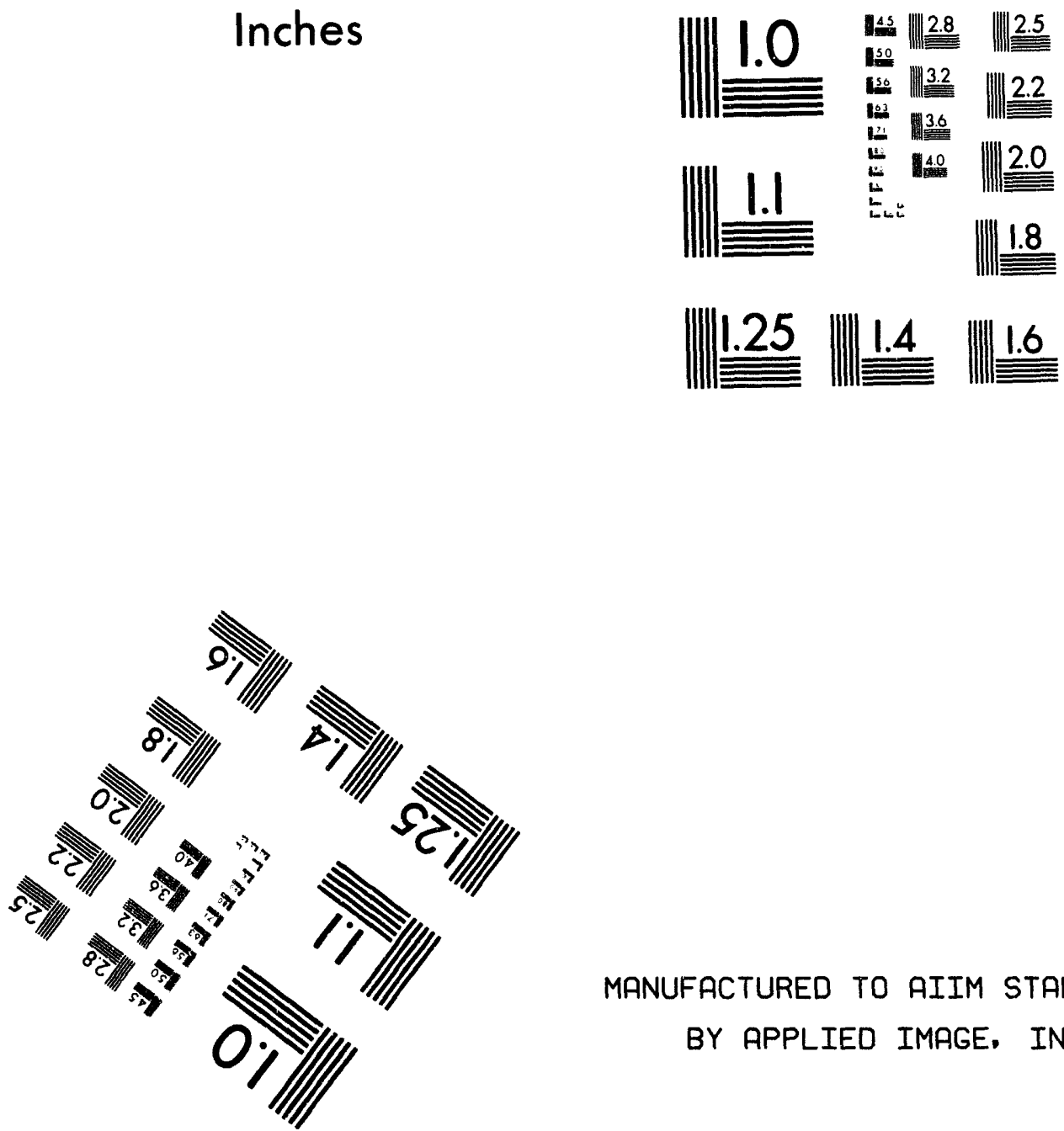

MANUFACTURED TO AIIM STANDARDS

BY APPLIED IMAGE, INC.

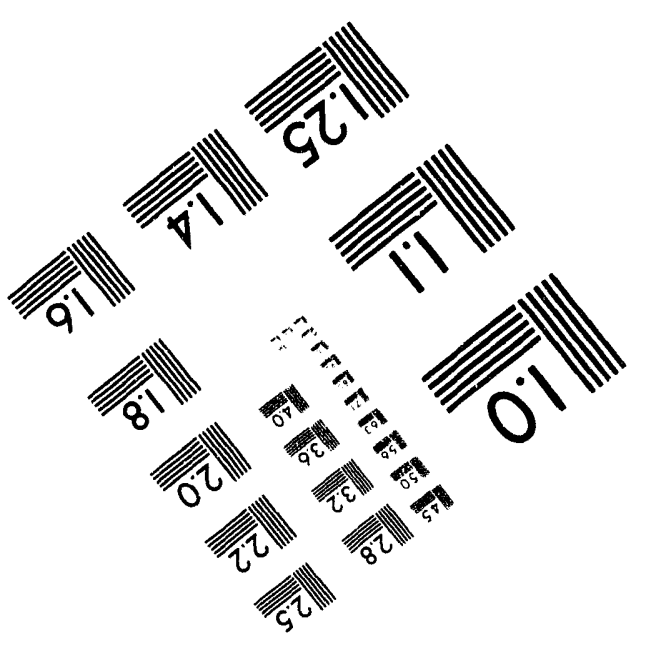



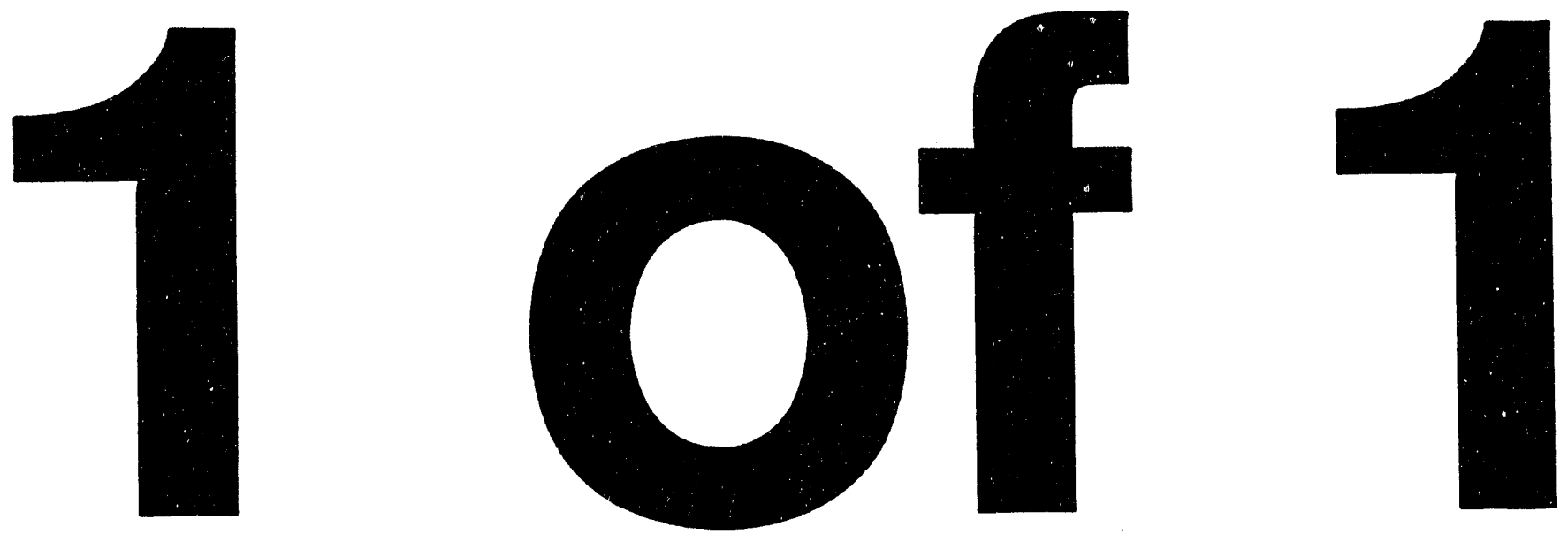


\title{
ANL/ATSD/CP. 83799
}

\section{Conf- $940701--18$}

Power-law resistivity, magnetic relaxation and ac susceptibility*

\author{
J. Gilchrist ${ }^{\mathrm{a}}$ and C.J. van der Beek \\ ${ }^{a}$ Centre de Recherches sur les Tres Basses Temperatures, laboratoire associe a I'Universite \\ Joseph Fourier, CNRS, BP 166, 38042 Grenoble-Cedex 9, France \\ bMaterials Science Division, Argonne National Laboratory, Argonne, Illinois 60439
}
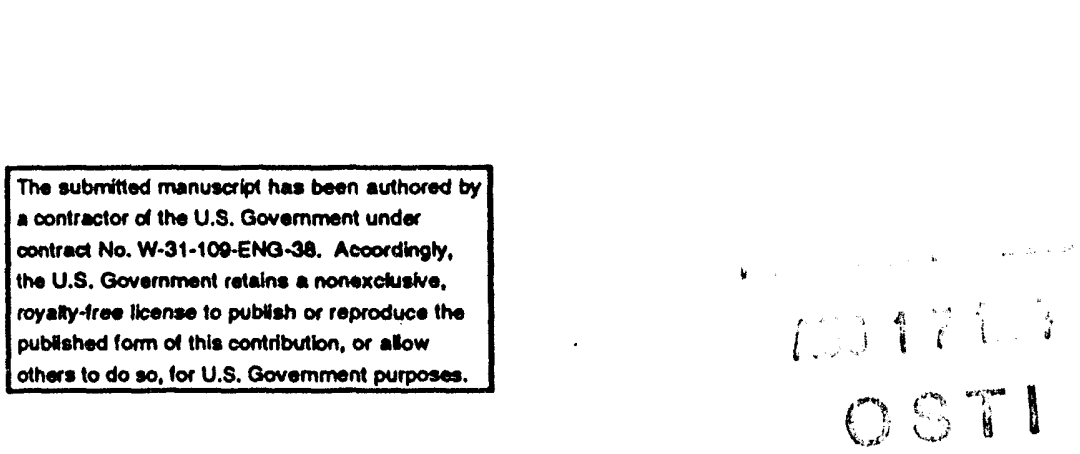

4th International Conference on Materials and Mechanisms of Superconductivity, High Temperature Superconductors (M²S-HTSC IV), Grenoble, France, July 5-9, 1994 (to be published in Physica C)

\section{DISCLAIMER}

\begin{abstract}
This report was prepared as an account of work sponsored by an agency of the United States Government. Neither the United States Government nor any agency thereof, nor any of their employees, makes any warranty, express or implied, or assumes any legal liability or responsibility for the accuracy, completeness, or usefulness of any information, apparatus, product, or process disclosed, or represents that its use would not infringe privately owned rights. Reference herein to any specific commercial product, process, or service by trade name, trademark, manufacturer, or otherwise does not necessarily constitute or imply its endorsement, recommendation, or favoring by the United States Government or any agency thereof. The views and opinions of authors expressed herein do not necessarily state or reflect those of the United States Government or any agency thereof.
\end{abstract}

\footnotetext{
*Work supported by the U.S. Department of Energy, BES-Materials Sciences under contract \#W-31-109-ENG-38.
} 
Power-law resistivity, magnetic relaxation and ac susceptibility

J. Gilchrista and C.J. van der Beek ${ }^{b}$

aCentre de Recherches sur les Très Basses Températures, laboratoire associé à l'Université Joseph Fourier, CNRS, BP 166, 38042 Grenoble-Cédex 9, France

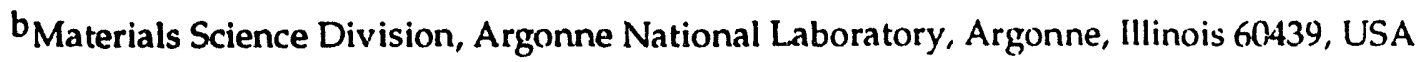

The nonlinear diffusion of magnetic flux into a superconducting sample can be studied by measuring the relaxation of the magnetisation after application of a step field or by measuring the ac sus ceptibility, $\chi_{1}$ and its third harmonic, $\chi_{3}$, or preferably both methods covering different time scales. Each has been analysed recently for a field-cooled sample of a material whose creep activation energy depends logarithmically on current density, J corresponding to a power-law relation between electric field, E and J. Here, results are compared, using a universal scaling depth. Maximum $\chi_{1}$ " and $\left|\chi_{3}\right|$ values occur, and also the magnetisation has relaxed to half its initial value when the scaling depth is comparable to the sample half-thickness.

Non-linear diffusion is a topic of wide general interest in physics (hydrodynamics, interface growth, chemical reactions, fractals, selforganised criticality). Magnetic flux diffuses non-linearly in a superconductor exhibiting flux creep and $a$ high $T_{c}$ material might provide a good practical example. To avoid unnecessary complications a monocrystalline platelet would be needed, with the applied field in its plane. The width, transverse to the field would need to be so much greater than the thickness as to overcome the intrinsic anisotropy of the material and allow the flux to penetrate from the broad faces rather than the edges. Bearing in mind these limitations, it is particularly profitable to analyse the case of a power-law $E(J)$, as Vinokur et al did [1], assuming for simplicity a field-cooled sample with prior existing flux density $B_{0}\left(\gg \mu_{0} H_{C_{1}}\right)$ and an increment $\delta B$ $\left(\ll B_{o}\right)$ caused by switching on an additional field. Numerical work [2] has shown that the results are qualitatively similar if an exponential $E(J)$ law is assumed. The analogous problem with an alternating perturbation of amplitude $\delta B$ has only been solved numerically $[3,4]$.
Flux creep consists of a series of hops (of bundles or individual lines) and a complete analysis would be a stochastic one. In the references cited [1-4], $E(J)$ was combined with Maxwell's equations to obiain a deterministic differential equation, and effectively the material, and the flux-line lattice treated as a continuum. This limits the validity to space and time scales large compared with the elementary hopping distance or hopping time, ignores noise and disallows any elastic response.

Putting $E=\mid \mathrm{J} / \mathrm{J} d{ }^{\sigma} \rho \mathrm{J}$ introduces two independent constants, the index $\sigma$ and the coefficient $J_{c}{ }^{-} \sigma_{\rho} . \rho$ is equivalent to $B u \omega_{m} / J_{c}$ of ref. 1 , but only the product $\mathrm{J}_{c}{ }^{-\sigma_{\rho}}$ is relevant to the flux diffusion problem. This $E(J)$ law evidently interpolates between Ohm's $(\sigma=0)$ and Bean's $(\sigma \rightarrow \infty)$.

There are basic similarities between the step field and the alternating field problems. For all $\sigma>0$ the perturbation is totally extinguished at a finite depth, $\delta_{e x}$, which depends on its amplitude and duration (or period). Two cases arise according to whether or not $\delta_{e x}$ exceeds the sample half-thickness, h. The extinction is 
abrupt if $\sigma>1$ but more gradual for low $\sigma$ values, so that $\delta_{e x}$ does not diways describe thes amount of flux penetration accurately. It is preferable to use a scaling lepth, $\bar{\delta}_{s}$ which, for an alternating field is a hybrid between Bean's penetration depth and the Ohmic skin depth:

$\delta_{s}^{2+\sigma}=\left(\delta B / \mu_{o} J_{c}\right)^{\sigma} 2 \rho / \mu_{o} \omega$

For the step field, $\omega^{-1}$ should be replaced by the time, $t$ since switch-on. Following Vinokur's analysis, but the present definition of $\delta_{s}$, the ratio $\delta_{\mathrm{ex}} / \delta_{\mathrm{s}}$ is expressible using $\Gamma$-functions, and is given for representative $\sigma$ values in Table 1. Also given is the ratio of sample half-thickness to scaling depth at the moment when the magnetisation has fallen to half the value, $M(0)$ it would in principle have had immediately after $\delta B$ was switched on. These values were obtained using a new numerical calculation and

\section{Table 1}

Ratio of total extinction depth to the time dependent scaling depth and ratio of sample halfthickness to scaling depth at the moment when the magnetisation has fallen to half its initial value. At this moment the whole sample has been penetrated, and $\delta_{e x} \geq h$.

\begin{tabular}{ccc}
\hline$\sigma$ & $\frac{\delta_{\text {ex }}}{\delta_{s}}$ & $\frac{h}{\delta_{c}}$ for $\frac{M(t)}{M(0)}=\frac{1}{2}$ \\
\hline 0 & $\infty$ & 1.59 \\
1 & 2.08 & 1.54 \\
2 & 1.77 & 1.49 \\
4 & 1.53 & 1.39 \\
6 & 1.42 & 1.33 \\
10 & 1.30 & 1.25 \\
$\infty$ & 1.00 & 1.00 \\
\hline
\end{tabular}

checked by an approximate analysis. In practice $S B$ is more lihely to be switched on with a finite ramp rate and M(l) deduced by extrapolation. The important point is that when $M(t) / M(0)=$ $1 / 2, h / \delta_{s}$ is always of order unity, which demonstrates that $\delta_{s}$ is a good measure of the flux penetration. $\sigma$ could be obtained from the limiting slope $S_{t \rightarrow \infty}=-1 / \sigma$, where $S=$ $\mathrm{d}(\ln \mathrm{M}) / \mathrm{d}(\ln t)[1] . \mathrm{J}_{\mathrm{C}}-\sigma_{\rho}$ could then be deduced using table 1 .

If an alternating $\delta B$ is used, the same information is obtainable by noting when $\chi_{1}^{\prime}=$ $-1 / 2$ (where the fundamental susceptibility is $\left.\chi_{1}^{\prime}-i \chi_{1}{ }^{\prime \prime}\right)$, or when $\chi_{1}$ " or $\left|\chi_{3}\right|$ is maximum. As Table 2 shows, all these conditions occur when $\mathrm{h} / \delta_{\mathrm{s}}$ is of order unity. If the third harmonic is used, a $\sigma$ value cannot be extracted reliably from $\left|\chi_{3}\right|_{\text {max }}$ as this tends to be lower than the theoretical value on account of edge effects and nonuniformities. Preferably the amplitude $\delta \mathrm{B}$ for $\left|\chi_{3}\right|_{\text {max }}$ should be found at several frequencies and the scaling law (Eq.1) used.

\section{REFERENCES}

1. V.M. Vinokur, M.V. Feigel'man and V.B. Geshkenbein, Phys. Rev. Lett. 67 (1991) 915 ; H.G. Schnack and R. Griessen, ibid. 68 (1992) 2706 ; V.M. Vinokur and V.B. Geshkenbein, ibid 68 (1992) 2707.

2. C.J. van der Beek, G.J. Nieuwenhuys, P.H. Kes, H.G. Schnack and R. Griessen, Physica C 197 (1992) 320.

3. J. Rhyner, Physica C 212 (1993) 292.

4. J. Gilchrist and T. Dombre, Phys. Rev. B 49 (1994) 1466.

Table 2

The analogue to table 1 for the case of an alternating applied field component. Except for $\sigma=0$ and $\infty$ the data all come from ref. 4. Rationalised units are used in which $\chi_{1}$ varies between -1 and 0 . Each of the three landmarks occurs where $\delta_{0 x} \geq h$ and the whole sample is penetrated.

\begin{tabular}{|c|c|c|c|c|c|c|}
\hline$\sigma$ & $\frac{\delta_{\text {ex }}}{\delta_{s}}$ & $\frac{h}{\delta_{s}}$ for $\chi_{1}^{\prime}=-\frac{1}{2}$ & $\chi_{1}{ }^{\prime \prime} \max$ & $\frac{h}{\delta_{s}}$ for $\chi_{1}{ }^{\prime \prime} \max$ & $\left|x_{3}\right|_{\max }$ & $\frac{h}{\delta_{s}}$ for $\left|\chi_{3}\right|_{\max }$ \\
\hline 0 & $\infty$ & 1.25 & 0.417 & 1.13 & $0 .(X)$ & +1 \\
\hline 1 & 3.49 & 1.22 & 0.375 & 1.06 & 0.0295 & 0.79 \\
\hline 3 & 2.00 & 1.18 & 0.340 & 1.01 & $0 .(470)$ & (1.74 \\
\hline 7 & 1.50 & 1.12 & 0.305 & 0.95 & $0 .(15+5$ & 0.70 \\
\hline$\infty$ & 1.00 & $1 .(x)$ & 0.239 & ().75 & $0 .(1613$ & (1.59 \\
\hline
\end{tabular}



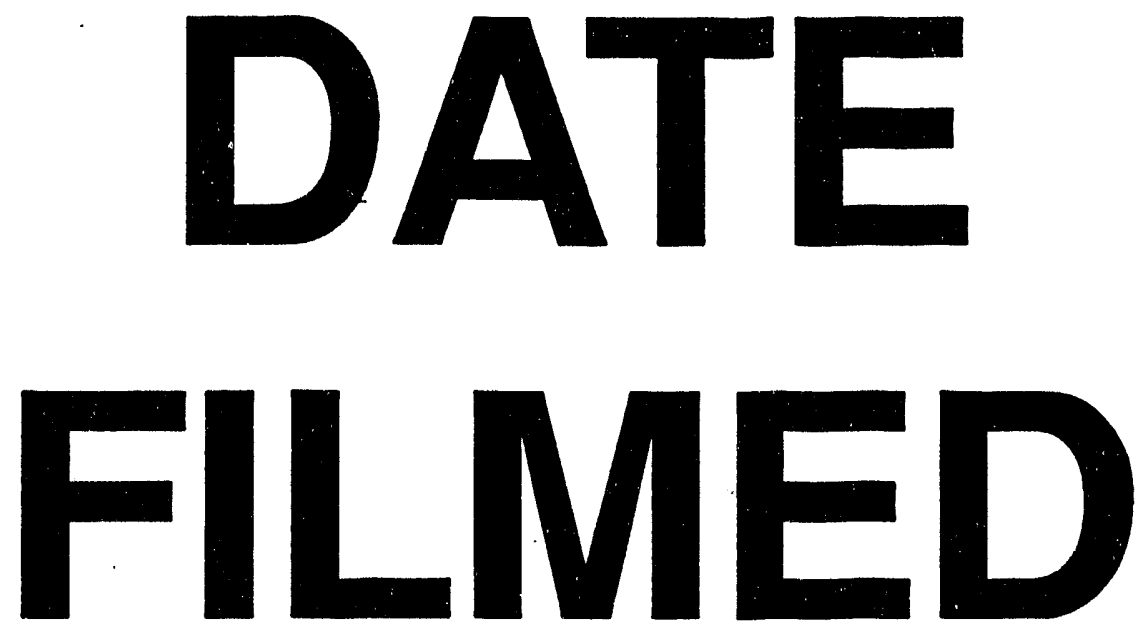

$10 / 13 / 94$
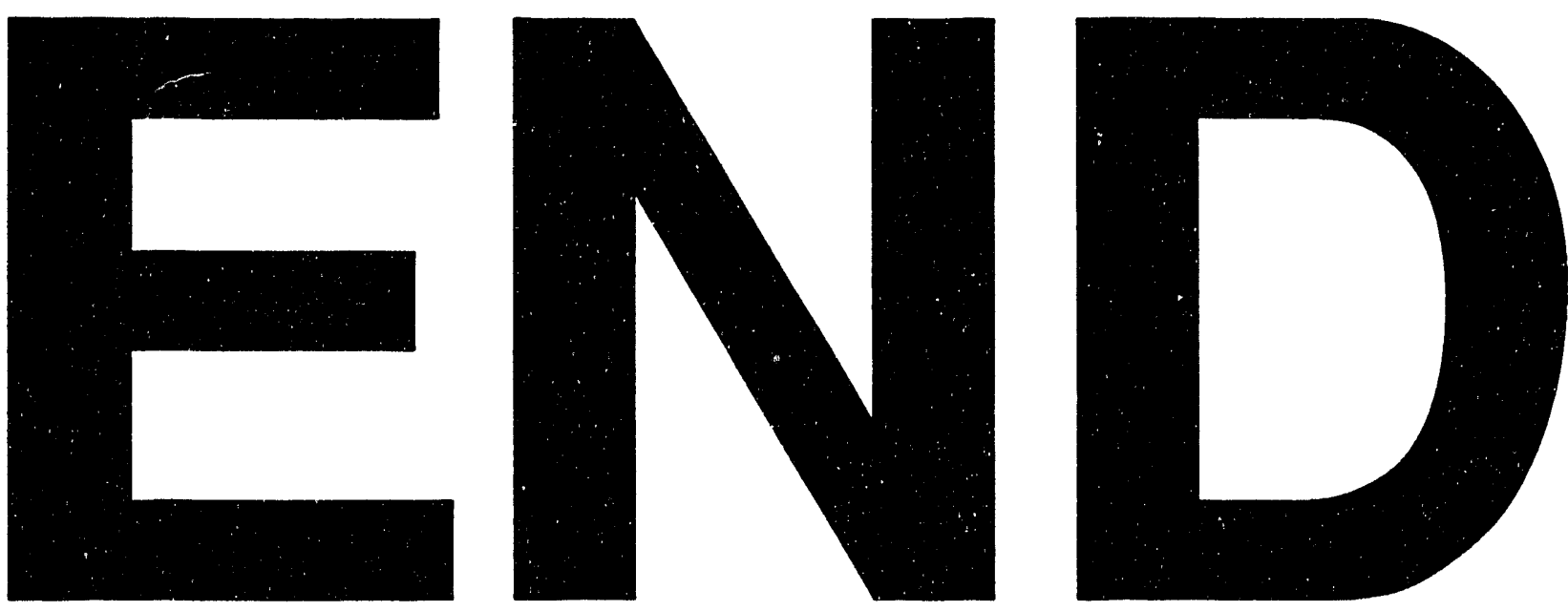
